\title{
Modeling Passive Mode-Locking in Quantum Dot Lasers: A Comparison Between a Finite- Difference Traveling-Wave Model and a Delayed Differential Equation Approach
}

\author{
Mattia Rossetti, Paolo Bardella, and Ivo Montrosset, Member, IEEE
}

\begin{abstract}
We present a detailed quantitative comparison between a finite-difference traveling wave (FDTW) model and a delayed differential equation (DDE) approach for the simulation of passive mode-locking in quantum dot lasers with both ring and Fabry-Perot (FP) cavities. Modifications with respect to the standard DDE models available in the literature are proposed. The new DDE approach improves the quantitative agreement with the FDTW model when applied to the simulation of passive mode-locking in FP lasers, preserving a very high computational efficiency. The modifications proposed in the DDE model also apply to the simulation of quantum-well and bulk devices.
\end{abstract}

Index Terms-Mode-locked lasers, quantum dots, semiconductor device modeling, semiconductor lasers.

\section{INTRODUCTION}

$\mathbf{P}$ ASSIVE mode-locking in multi-section quantum-dot (QD) lasers has been extensively studied in the last years both experimentally and theoretically due to their expected superior properties with respect to bulk and quantum-well counterparts [1], [2]. From the theoretical point of view, different numerical models have been proposed allowing to gain insights on the physical mechanisms governing the onset of the ML regimes. Finite-difference travelling-wave (FDTW) models allow for an accurate description of these devices; this approach is based on the direct solution via a finitedifference method of the one-dimensional first-order waveequations governing the field evolution inside the cavity; these equations are then coupled, via proper polarization terms, with a set of rate equations modelling carrier dynamics within the QD states in each longitudinal section of the cavity [3], [4]. FDTW models allow to accurately describe two-section QD passively mode-locked lasers with both ring and Fabry-Perot (FP) cavities.

Alternatively, assuming a unidirectional ring cavity and introducing suitable approximations, one can derive from the

Manuscript received October 28, 2010; revised December 21, 2010; accepted December 26, 2010. Date of current version March 25, 2011. This work was supported in part by the European Community's Seventh Framework Program FP7/2007-2013 under Grant Agreement 224338 (FASTDOT project).

The authors are with the Dipartimento di Elettronica, Politecnico di Torino, Turin 10129, Italy (e-mail: mattia.rossetti@ polito.it; paolo.bardella@ polito.it; ivo.montrosset@polito.it).

Color versions of one or more of the figures in this paper are available online at http://ieeexplore.ieee.org.

Digital Object Identifier 10.1109/JQE.2010.2104135 fundamental wave-equations an alternative set of equations describing the laser dynamics; these equations are based on a delayed differential equation (DDE) coupled to rate equations governing the carrier dynamics in the gain and saturable absorber (SA) sections. The DDE model is essentially a generalization of New and Haus analytical models [5], [6]. It was originally formulated by Vladimirov et al. in [7], [8] and successively extended to the analysis of QD ML lasers [9]. This approach has the advantage of an extremely reduced computational cost with respect to the FDTW models; moreover, taking advantage of its simplicity, the DDE model allows for both analytical and numerical studies of the bifurcations leading to different dynamic regimes. This approach has been extensively applied to study passive ML in QD FP lasers [10]-[12] even if the model would be strictly valid for unidirectional ring lasers only. A qualitative agreement between DDE and FDTW models when simulating FP QD ML lasers has been demonstrated [12], [13].

This work establishes a quantitative comparison between a FDTW model and a DDE approach for the simulation of ML in QD lasers with both ring and FP cavities. In order to improve the agreement, we propose a generalization of the DDE approach [7]-[12] considering a discretization in subsections of the gain and saturable absorber sections. We show that, with respect to standard DDE approaches [7]-[12], the proposed multi-section DDE (MS-DDE) model allows for an improved quantitative agreement with the FDTW model when simulating a QD FP ML laser.

To establish a quantitative comparison, in the MS-DDE model, input parameters for the description of carrier dynamics in the QD material are kept the same as those in the FDTW model; moreover, the FDTW model is implemented with a formulation as close as possible to that used in the MS-DDE approach.

It must be pointed out that the modifications introduced in the MS-DDE model remain valid and effective even when rate equations describing carrier dynamics in quantum-well and bulk active media are considered.

In section II, the implemented FDTW model for QD ML lasers is described; the developed multi-section DDE model is presented and the differences with respect to standard DDE approaches available in the literature are highlighted. As a first validation, in section III, the two numerical models are applied 
to the simulations of QD ML lasers with ring cavity geometry. In section IV, the two approaches are then used for the simulations of FP ML lasers. A detailed comparison is established, demonstrating that the additional features introduced in our multi-section DDE model allow for an improved agreement with the FDTW model when simulating QD FP ML lasers. Finally a brief conclusion is drawn.

\section{Numerical Models}

\section{A. Finite Difference Travelling-Wave Model}

The amplitude of the guided mode travelling along the longitudinal direction $(z)$ of a FP laser cavity can be written in terms of forward and backward propagating envelopes $E_{i}^{ \pm}(z, t)$ which are slowly varying both in time and space:

$$
\Psi_{i}(z, t)=\left(E_{i}^{+}(t, z) e^{-j k_{i} z}+E_{i}^{-}(t, z) e^{+j k_{i} z}\right) e^{j \omega_{i} t}
$$

where $\omega_{i}$ is a reference frequency chosen close to the lasing frequency, $k_{i}=\omega_{i} / v_{g}$ is the corresponding reference wavenumber, where $v_{g}$ is the group velocity. Index $i=G S, E S_{1}$ indicates the field component which is resonant either with the QD ground states (GS) or the QD first excited state $\left(E S_{1}\right)$. The spatiotemporal evolution of each forward and backward travelling GS and $\mathrm{ES}_{1}$ field envelopes is described by a dedicated first-order wave equation as follows:

$$
\begin{aligned}
& \frac{1}{v_{g}} \frac{\partial E_{G S}^{ \pm}}{\partial t} \pm \frac{\partial E_{G S}^{ \pm}}{\partial z}=\Gamma_{x y} g_{G S}(t, z)\left(\gamma e^{-\gamma t} \otimes E_{G S}^{ \pm}(t, z)\right) \\
& \quad+j \beta \Gamma_{x y} g_{E S_{1}}(t, z) E_{G S}^{ \pm}(t, z)-\frac{\alpha_{i}}{2} E_{G S}^{ \pm}(t, z) ; \\
& \frac{1}{v_{g}} \frac{\partial E_{E S_{1}}^{ \pm}}{\partial t} \pm \frac{\partial E_{E S_{1}}^{ \pm}}{\partial z}=\Gamma_{x y} g_{E S_{1}}(t, z)\left(\gamma e^{-\gamma t} \otimes E_{E S_{1}}^{ \pm}(t, z)\right) \\
& \quad-j \beta \Gamma_{x y} g_{G S}(t, z) E_{E S_{1}}^{ \pm}(t, z)-\frac{\alpha_{i}}{2} E_{E S_{1}}^{ \pm}(t, z) ; \quad \text { (2b) }
\end{aligned}
$$

$g_{i}(t, z)$ represents the QD material gain at wavelength resonant with $i=G S, E S_{1} ; g_{i}(t, z)$ is time and space dependent and its dynamics is computed via a suitable set of rate equations, $\Gamma_{x y}$ is the field confinement factor within the QD layers and $\alpha_{i}$ are the intrinsic waveguide losses. $\otimes$ stands for a convolution product which describes the field filtered by a Lorentzian function of width $\gamma$, modelling the finite QD gain spectral bandwidth which is limited by the QD inhomogeneous gain broadening. In the second term in the RHS of (2a) and (2b), $\beta \Gamma_{x y} g_{G S}(t, z)$ and $\beta \Gamma_{x y} g_{E S}(t, z)$ represent instant changes in the real part of the propagation constant, induced by the QD ES at the GS wavelength and vice versa; these contributions are in fact the main responsible for a non-zero chirp of the ML pulses due to self-phase modulation [14]. $\beta$ is a coefficient which depends on the frequency separation between QD GS and $\mathrm{ES}_{1}$ and can be approximated as:

$$
\beta=\gamma \frac{\omega_{E S_{1}}-\omega_{G S}}{\gamma^{2}+\left(\omega_{E S_{1}}-\omega_{G S}\right)^{2}} .
$$

Wave equations (2) are then completed with proper boundary conditions that in the case of a FP laser cavity can be written as:

$$
\begin{aligned}
E_{i}^{+}(t, 0) & =\sqrt{R_{1}} E_{i}^{-}(t, 0) ; \\
E_{i}^{-}\left(t, L^{F P}\right) & =\sqrt{R_{2}} E_{i}^{+}\left(t, L^{F P}\right) ; i=G S, E S_{1}
\end{aligned}
$$

where $L^{F P}$ is the total FP cavity length and $R_{1}$ and $R_{2}$ are the power reflectivities at the device facets. The output power will be therefore simply given by $P_{1 i}=\left(1-R_{1}\right)\left|E_{i}^{-}(t, 0)\right|^{2}$ and $P_{2 i}=\left(1-R_{2}\right)\left|E_{i}^{+}\left(t, L^{F P}\right)\right|^{2}$.

As already pointed out, in order to compute the gain/absorption dynamics in each longitudinal section of the cavity, a proper set of rate equations is solved. This set consists of 3 rate equations to describe carrier dynamics in 3 confined QD states (GS, $\left.\mathrm{ES}_{1}, \mathrm{ES}_{2}\right)$ in the excitonic approach and 2 additional rate equations for carrier densities in the quantumwell (QW) and in the barrier states, respectively. The rateequations can properly describe the population dynamics in the QD under both current injection and reverse bias conditions [3]. Modified rate equations have been therefore introduced in order to model the population dynamics in the QD belonging to SA section, where voltage dependent thermionic escape and tunnelling processes are taken into account [3].

The rate equations for the QD GS and $\mathrm{ES}_{1}$ populations have the form:

$$
\frac{d \rho_{i}(t, z)}{d t}=R_{i}^{\text {in }}(t, z)-R_{i}^{\text {out }}(t, z)-R_{i}^{s}(t, z)-R_{i}^{\text {stim }}(t, z)
$$

where $\rho_{i}(t, z)$ represents the occupation probability in the $i^{t h}$ QD state in the cavity section z. $R_{i}^{\text {in }}(t, z)$ and $R_{i}^{\text {out }}(t, z)$ are in and out scattering rates, $R_{i}^{s}(t, z)$ represents the radiative and non radiative recombination rate. A detailed description of the rate equations system containing explicit expressions for the rates appearing in (5) can be found in [3]. Finally $R_{i}^{\text {stim }}(t, z)$ represents the stimulated emission rate that can be written as follows:

$$
\begin{aligned}
R_{i}^{s t i m}(t, z)= & \frac{2 \Gamma_{x y} g_{i}(t, z)}{W N_{D} D_{i} \hbar \omega_{i}} \\
& \times \sum_{ \pm} \Re e\left[E_{i}^{ \pm *}(t, z) \cdot\left(\gamma e^{-\gamma t} \otimes E_{i}^{ \pm}(t, z)\right)\right]
\end{aligned}
$$

where $g_{i}(z, t)=g_{0 i}\left(2 \rho_{i}(t, z)-1\right), W$ is the ridge width, $N_{D}$ is the QD surface density and $D_{i}$ is the degeneracy of the $\mathrm{i}^{\text {th }}$ QD state. Finally $g_{0 i}$ is the material gain coefficient for the $\mathrm{i}^{\text {th }}$ QD state.

Equations (2)-(6) can therefore be solved directly using a finite difference approach, allowing to compute the spatiotemporal dynamics of the field along the longitudinal direction of the FP cavity. A temporal grid with time step $\Delta t=30 \mathrm{fs}$ and a spatial grid with unit step $\Delta z=v_{g} \cdot \Delta t$ is considered in the finite difference scheme.

In the case of a unidirectional ring cavity laser, similar equations can be used: since propagation is assumed to occur in a unique direction, the optical field can be described by a single slowly varying envelope, e.g. $E_{i}^{+}(t, z)$ with $i=G S, E S_{1}$. The counter-clockwise field will be therefore set to be identically zero $E_{i}^{-}(t, z)=0$.

In this way the dynamics of $E_{i}^{+}(t, z)$ can be simply computed by using (2a). Moreover, (4) can be simply substituted by the following boundary condition:

$$
E_{i}^{+}(t, 0)=\sqrt{K} E_{i}^{+}\left(t, L^{\text {Ring }}\right)
$$

where $K$ are the losses induced by the output coupling localized in $z=0$. 


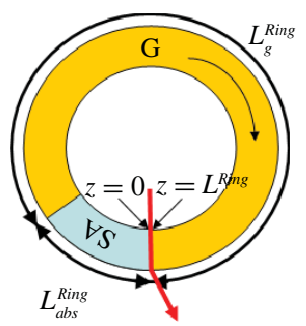

(a)

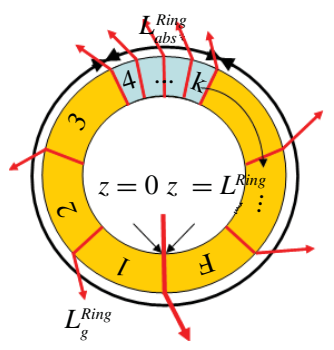

(c)

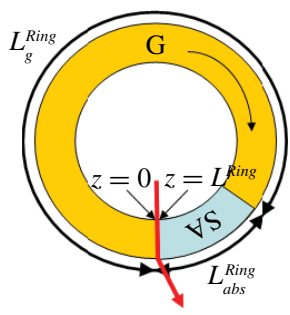

(b)

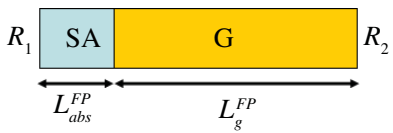

(d)
Fig. 1. (a) Schematic of unidirectional ring laser described by a standard DDE model [7]-[12]; red line and arrow indicate nonsaturable losses localized in the reference section of the device. (b) Alternative ring laser structure that can be described by a standard DDE approach. (c) Schematic of ring laser that can be described by the proposed MS-DDE model; the device consists of F isolated sections; red lines/arrows indicate nonsaturable losses introduced at any interface between two adjacent sections. (d) Schematic of a two section FP ML laser. Forward biased gain sections (G) (yellow) and reverse biased saturable absorber sections (SA) (light blue) are also highlighted.

The described approach differs from [3] in the description of the QD active medium. Here the inhomogeneous gain broadening induced by the QD size dispersion is not considered to allow for a direct comparison with the DDE model described in the next section.

\section{B. Multi-Section Delayed Differential Equation Model}

The described FDTW model has, as a main drawback, the high computational cost required by the simulations, caused by the dense spatial and temporal discretization in the finitedifference scheme. The DDE model [7]-[12] on the contrary represents a simple and computationally efficient approach for the dynamic simulation of such devices.

The DDE equations governing the field dynamics in the laser cavity have been directly derived from the original wave equations (2) but several assumptions have been introduced. In the DDE model proposed in [7], [8] and extended to the simulation of QD lasers in [9], the following hypotheses have been assumed:

1) A ring cavity with unidirectional lasing;

2) Filtering effect due to the finite gain spectral bandwidth is modelled as a lumped element in a single reference section of the device $(z=0)$;

3) Intrinsic waveguide losses $\alpha_{i}$ are treated together with the output coupling losses as lumped losses localized in the same reference section of the device $(z=0)$;

4) The ring laser is assumed to consist of only two sections: the saturable absorber (SA) and the gain (G) section;

5) Lasing from the QD GS only is assumed.
A schematic of the structure considered in [7]-[12] is shown in Fig. 1a.

In this paper we propose instead a generalization of the DDE model proposed in [9]-[12] where assumptions 1 and 2 in the above list still apply but:

1) The ring laser is assumed to be composed by an arbitrary number of electrically isolated sections that can be independently forward or reverse biased;

2) The non saturable loss terms are introduced between any two adjacent sections modelling more accurately the effect of intrinsic waveguide losses and output coupling losses.

3) Lasing from both QD GS and $\mathrm{ES}_{1}$ can be properly treated.

With respect to previous approaches, our MS-DDE model allows to describe the structure as depicted in Fig. 1c.

DDE equations governing the evolution of the field at both GS and ES1 wavelengths as well as the population dynamics in the QD states belonging to each independent section of the device can be directly derived from (2a), (3), (5) and (7). By considering the following coordinate change $(t, z) \rightarrow(\tau=t-$ $\left.z / v_{g}, z\right)$ and following essentially the same derivation as in [7], we end up with the set of equations described below. Being $F$ the total number of electrically isolated sections in the device and being $L_{k}$ the length of the $\mathrm{k}^{t h}$ section $(k=1,2, \ldots F)$, the following DDE equations can be derived:

$\frac{d E_{i}(\tau)}{d \tau}=-\gamma E_{i}(\tau)+\gamma R_{i}(\tau-T) E_{i}(\tau-T) \quad i=G S, E S_{1}$

where $E_{i}(\tau)=E_{i}^{+}(\tau, 0)$ is the field in the reference section $z=0$ resonant with $G S$ and $E S_{1}$ wavelengths, respectively; $T$ is the cold cavity round trip time given by $T=L^{\text {Ring }} / v_{g}$ with $L^{\text {Ring }}=\sum L_{k}$ and $R_{i}(\tau)$ represents the round-trip gain experienced by the pulse within the cavity. $R_{i}(\tau)$ can be simply computed via the following expression:

$$
R_{i}(\tau)=\prod_{k=1}^{F} B_{i k}(\tau) M_{k}
$$

In (9), $M_{k}$ describes the non saturable losses localized between the $k^{\text {th }}$ and $(k+1)^{\text {th }}$ section. This term can include intrinsic waveguide losses experienced by the field when travelling across the $k^{t h}$ section i.e. $\exp \left(-\alpha_{i} / 2 \cdot \mathrm{L}_{k}\right)$ and output coupling losses $\sqrt{K_{k}}$ localized at the interface between the two sections:

$$
M_{k}=\sqrt{K_{k}} e^{-\alpha_{i} / 2 L_{k}}
$$

$B_{i k}(\tau)$ represents instead the amplification or attenuation and the phase changes experienced by the field at $i=G S, E S_{1}$ wavelength when travelling across the $k^{t h}$ section. Those terms can be simply written as:

$$
\begin{aligned}
B_{G S, k}(\tau)= & \exp \left(\Gamma_{x y} \bar{g}_{G S, k}(\tau) L_{k}\right) \\
& \times \exp \left(j \beta \Gamma_{x y} \bar{g}_{E S_{1}, k}(\tau) L_{k}\right) \\
B_{E S_{1}, k}(\tau)= & \exp \left(\Gamma_{x y} \bar{g}_{E S_{1}, k}(\tau) L_{k}\right) \\
& \times \exp \left(-j \beta \Gamma_{x y} \bar{g}_{G S, k}(\tau) L_{k}\right)
\end{aligned}
$$

where $\bar{g}_{i, k}(\tau)$ represents the average gain/absorption induced by the QD GS or $\mathrm{ES}_{1}$ in the $k^{\text {th }}$ section. This quantity can be 


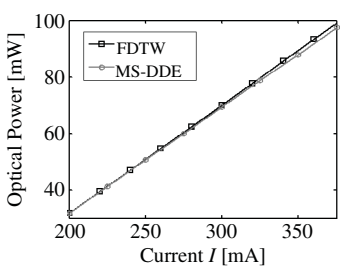

(a)

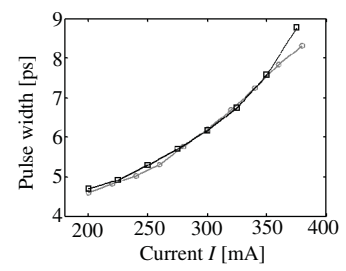

(c)

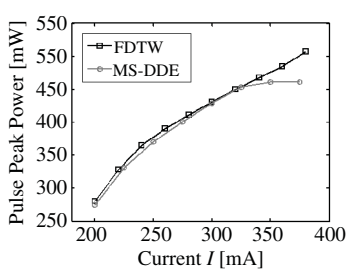

(b)

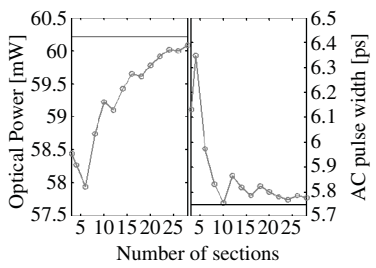

(d)
Fig. 2. $(a-c)$ Comparison between results obtained with the FDTW model (black lines, square markers) and the MS-DDE model (gray lines, circle markers) from the simulation of mode-locking in the ring laser structure depicted in Fig. 1(c). The behavior of average power (a), pulse peak power (b), and pulse width (c) is shown as a function of the current applied to the gain section, for a fixed $-7 \mathrm{~V}$ SA reverse bias. (d) Average power and pulse width computed using the MS-DDE model for $V=7 \mathrm{~V}$ and $I=275 \mathrm{~mA}$ as a function of the number of sections $F$ used in the calculation. Black lines show the corresponding values obtained using the FDTW model.

calculated as:

$$
\bar{g}_{i, k}(\tau)=g_{0 i}\left(2 \bar{\rho}_{i, k}(\tau)-1\right) ;
$$

where $\bar{\rho}_{i, k}$ is the occupation probability in the $i^{\text {th }}$ QD state averaged over the $k^{\text {th }}$ section. The temporal dynamics of $\bar{\rho}_{i, k}$ in each section of the ring cavity can be computed by a system of rate equations which is almost identical to the one used in the FDTW model [3]:

$$
\frac{d \bar{\rho}_{i k}}{d \tau}=\bar{R}_{i k}^{\text {in }}(\tau)-\bar{R}_{i k}^{\text {out }}(\tau)-\bar{R}_{i k}^{\text {stim }}(\tau)
$$

apart from the stimulated emission rates that in this case can be written as follows:

$$
\begin{aligned}
\bar{R}_{i k}^{\text {stim }}(\tau)= & \left(\left|B_{i k}(\tau)\right|^{2}-1\right) \\
& \times\left(\prod_{m=1}^{k-1}\left|B_{i m}(\tau)\right|^{2} M_{m}^{2}\right) \frac{\left|E_{i}(\tau)\right|^{2}}{\hbar \omega_{i} D_{i} N_{D} W L_{k}} .
\end{aligned}
$$

Finally, the output power from section $\mathrm{z}=0$, is calculated as:

$$
P_{i}^{\text {out }}(\tau)=\frac{1-K_{F}}{K_{F}}\left|E_{i}(\tau)\right|^{2} .
$$

Equations (8)-(14) represent the complete set of differential equations to be solved in order to compute the temporal evolution of the field in the reference section $z=0$.

In the next sections, the FDTW model and the MS-DDE model described above will be applied to the simulation of both unidirectional ring lasers and FP ML lasers.

\section{Mode-Locking In IdEAL Unidirectional RinG LASERS}

We consider a unidirectional ring laser with total length $L^{\text {Ring }}=L_{g}^{\text {Ring }}+L_{a b s}^{\text {Ring }}=4 \mathrm{~mm}$ and SA length $L_{a b s}^{\text {Ring }}=$ $700 \mu \mathrm{m}$. The simulated device corresponds to the one depicted in Fig. 2c: a uniform current injection is assumed in the whole gain section so that, in the MS-DDE model, all the considered forward biased sections have the same injected current density. The losses due to output coupling are assumed to be located in the middle of the gain section in $z=0$ only. We set $K=0.1$, corresponding to $90 \%$ extraction, in the FDTW model; correspondingly we set $K_{F}=0.1$ and $K_{k}=1$ for $k=1 \ldots(F-1)$ in the MS-DDE approach. Intrinsic waveguide losses $\alpha_{i}=$ $1.5 \mathrm{~cm}^{-1}$ have been considered.

The QD active region is assumed to consist of 5 layers of InAs QDs embedded in a InGaAs quantum-well and emitting around $1.3 \mu \mathrm{m}$ [3]. Material parameters involved in the rateequations, describing the physical properties of the QD active medium, are fully consistent with parameters reported in [3]. Gain coefficients for QD GS and $\mathrm{ES}_{1}$ are $\Gamma_{x y} g_{0 G S}=$ $12.7 \mathrm{~cm}^{-1}$ and $\Gamma_{x y} g_{0 E S}=22.8 \mathrm{~cm}^{-1}$, respectively. The width of the Lorentzian filter $\gamma$ is set as the width of the inhomogeneous gain broadening induced by the QD size dispersion: $2 \hbar \gamma=34 \mathrm{meV}$.

Simulations of mode-locking in this structure have been performed with both the MS-DDE model and the FDTW model. A total number of sections $F=28$ composing the whole ring cavity has been chosen in the MS-DDE model.

We compare the ML regimes obtained with the two approaches for a fixed $7 \mathrm{~V}$ reverse bias applied to the SA section. In the whole range of investigated bias parameters, only GS lasing has been achieved. In Fig. 2, the behaviour of average power, peak power and pulse width (calculated through the intensity autocorrelation function of the ML pulses and assuming a Gaussian pulse shape) are shown as a function of the current applied to the gain section. A very good agreement between the two models has been achieved over a wide range of applied currents. Appreciable differences in the results have been obtained at very large currents only. We think that this discrepancy can be mainly attributed to the different treatment of the intrinsic waveguide losses in the FDTW (where they are distributed along the whole cavity) and in the MS-DDE model (where they are assumed to be localized between two adjacent sections only); as a matter of fact, this difference may lead to a different gain saturation induced by the pulse in the gain section and this discrepancy tends to become more relevant at high currents (high pulse energies). Fig. 2d shows the behaviour of average power and pulse width, simulated using the MS-DDE for a fixed 7V SA voltage and $275 \mathrm{~mA}$ gain current, as a function of the number of sections $F$ ranging from the minimum value $F=3$ up to $F=28$. This clearly shows that results obtained with the MS-DDE model remain rather accurate even for low number of sections.

Finally, in order to study the stability of the obtained ML regimes to perturbations induced by the spontaneous emission noise, we can calculate the net gain experienced by the ML pulse over a single round trip in the cavity. From the MLDDE model, the round trip gain experienced by the ML pulse is simply given by (9). Equation (9) can then be rewritten as follows:

$$
\left|R_{i}(\tau)\right|=\exp \left(\left(G_{i}(\tau)-A_{i}(\tau)\right) L^{\text {Ring }}\right) \quad i=G S, E S_{1}
$$




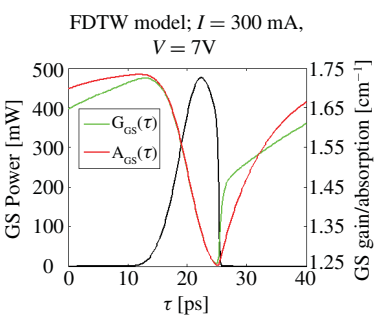

(a)

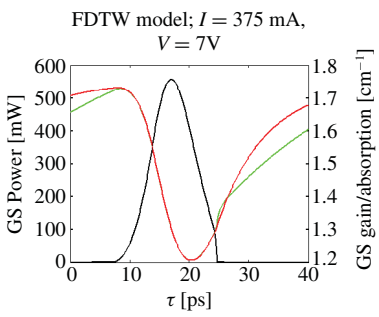

(c)

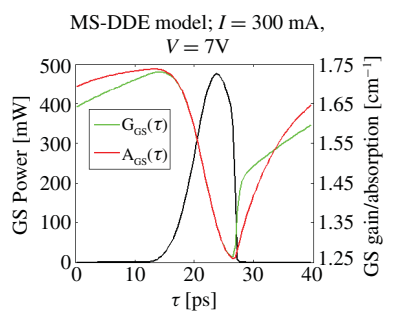

(b)

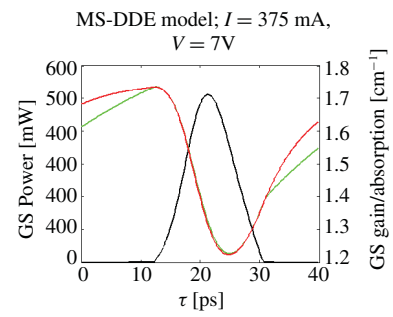

(d)
Fig. 3. Net gain windows leading to the onset of the ML regime in the ring laser structure: pulse time trace in $z=L^{\text {Ring }}$ (blue), $A_{G S}(\tau)$ (red), and $G_{G S}(\tau)$ (green) computed using the MS-DDE model (right) and the FDTW model (left), are shown for a 7-V SA reverse bias and gain currents of (a), (b) $300 \mathrm{~mA}$ and (c), (d) $375 \mathrm{~mA}$.

where $A_{i}(\tau)$ (in $\mathrm{cm}^{-1}$ ) represents the overall saturable and non saturable losses experienced by the ML pulse over a round trip whereas $G_{i}(\tau)$ (in $\mathrm{cm}^{-1}$ ) represents the overall amplification in the gain section. $A_{i}(\tau)$ and $G_{i}(\tau)$ can therefore be simply calculated as follows:

$$
\begin{aligned}
G_{i}(\tau)= & \frac{1}{L^{\text {Ring }}} \sum_{\substack{k \in \text { Gain } \\
\text { section }}} \log \left(\left|B_{i k}(\tau)\right|\right) ; \\
A_{i}(\tau)= & \frac{\alpha_{i}}{2}+\frac{1}{L^{\text {Ring }}} \\
& \times\left\{\sum_{k=1}^{F} \log \left(\frac{1}{\sqrt{K_{k}}}\right)-\sum_{\substack{k \in \mathrm{SA} \\
\text { section }}} \log \left(\left|B_{i k}(\tau)\right|\right)\right\}
\end{aligned}
$$

The same quantities can be computed from the FDTW model by integration over the spatial coordinate $\mathrm{z}$, as described in detail in [3]. In Fig. 3 we compare the behaviour of $A_{G S}(\tau)$ and $\mathrm{G}_{G S}(\tau)$ at a fixed 7V SA voltage and gain current equal to $300 \mathrm{~mA}$ and $375 \mathrm{~mA}$, obtained with the MS-DDE model (with $F=28$ ) and the TDTW model, respectively. A very good agreement between the two modelling approaches is obtained. As already reported in [3], at low currents, an ultrafast recovery of the GS population just after the ML pulse due to fast ES to GS transitions, leads to a corresponding steep increase in $\mathrm{G}_{G S}(\tau)$. This creates therefore a region of net gain $\mathrm{R}_{G S}(\tau)>1$ just after the pulse trailing edge. For current close to the laser threshold, the net gain window exceeding the pulse trailing edge becomes large enough so that spontaneous emission noise generated within this time interval can grow, inducing a trailing edge instability in the obtained ML regime.
Increasing the applied current, this region of net gain just after the pulse vanishes and, according to New's criteria [5], [6], [15], a region of perfectly stable mode-locking can be achieved for $I=375 \mathrm{~mA}$ as shown in Fig. 3b and Fig. 3d.

In this paragraph we have shown that the proposed MS-DDE approach can give results in good quantitative agreement with those obtained with the FDTW model, when used to simulate ML in QD unidirectional ring lasers. In the next section we will establish instead a comparison between results obtained with the MS-DDE model and the FDTW model when applied to the analysis of Fabry-Perot two-section ML lasers.

\section{Mode-Locking In FABRY-Perot LASERS}

Let consider a two-section FP laser as the one depicted in Fig. 1d. The device is supposed to have a total length $L^{F P}=2$ $\mathrm{mm}$, a SA length $L_{a b s}^{F P}=350 \mu \mathrm{m}$, a high-reflection (HR) coated facet with $R_{1}=0.99$ at the SA side and an antireflection (AR) coated output facet with $R_{2}=0.1$.

The FDTW model allows for an accurate description of such a structure, as described in section II. On the contrary, a DDE approach cannot in principle ensure an accurate description of FP lasers since a unidirectional ring cavity is strictly assumed.

In order to use a DDE model for the analysis of such devices, we have therefore to set $L^{\text {Ring }}=2 L^{F P}$, ensuring the same cold cavity round trip frequency in both structures and similarly: $L_{a b s}^{\text {Ring }}=2 L_{a b s}^{F P}$.

Furthermore we must ensure that the overall non-saturable losses experienced by the field over a round trip are the same in both structures. To satisfy this condition, in previous DDE models [7]-[12], the only possibility was to set the lumped attenuation factor $K$, localized at the reference section $z=0$, simply as $K=R_{1} R_{2} \exp \left(-\alpha_{i} L^{F P}\right)$. Moreover since in previous DDE approaches the whole ring cavity was assumed to consist of one SA section and one gain section only, the SA section was forced to be located just before or after the reference section $z=0$ where non-saturable losses were introduced, as schematically shown in Fig. 1a and Fig. 1b, respectively.

On the contrary, in the proposed MS-DDE model, the nonsaturable losses can be distributed between any two adjacent cavity sections. This allows to consider an equivalent ring laser structure as that shown in Fig. 2c, where coefficients $M_{k}$ defined in (9) and (10) are in this case chosen as:

$$
M_{k}=\left\{\begin{array}{l}
\sqrt{R_{2}} e^{-\alpha_{i} / 2 L_{F}} \quad \text { if } k=F \\
\sqrt{R_{1}} e^{-\alpha_{i} / 2 L_{F / 2}} \quad \text { if } k=F / 2 \\
e^{-\alpha_{i} / 2 L_{k}} \quad \text { if } k \neq F / 2 \text { and } k \neq F
\end{array}\right.
$$

with $F$ even.

The previous conditions ensures that, for a given current density injected in the gain section, the FP ML laser under study and the corresponding equivalent ring structure have the same total unsaturated round trip gain.

Let consider at first a uniform current injection in both the gain and SA section so that the device operates as a simple FP laser in continuous wave regime. In Fig. 4a, we compare the simulated light-current characteristic obtained with the FDTW model and the MS-DDE model, the latter applied to the 

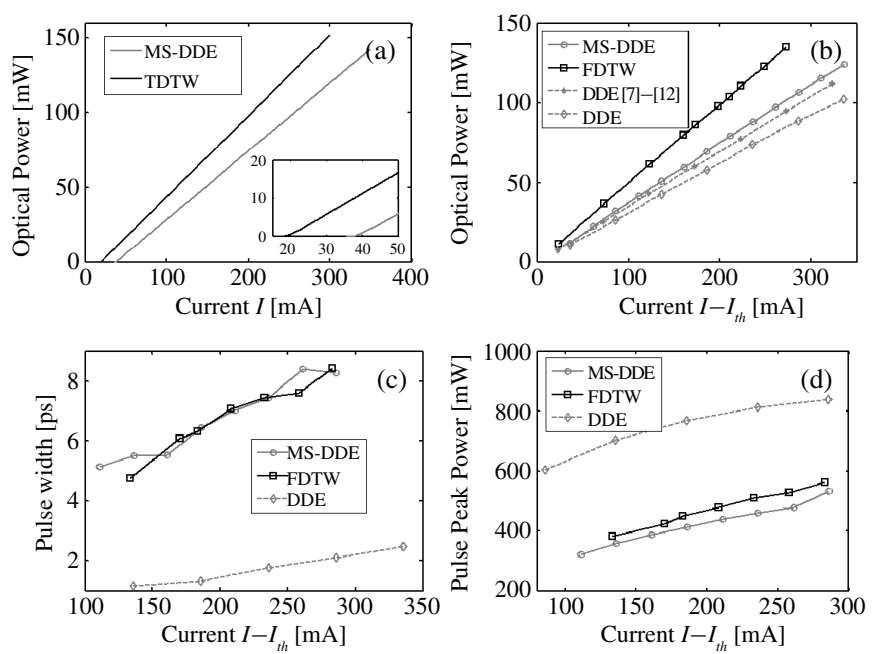

Fig. 4. (a) Comparison between the light-current characteristic obtained from the simulations of the FP laser with uniform injection using the FDTW model and the one obtained from the simulation of the equivalent ring laser structure using the MS-DDE model. Output power from the anti-reflection coated facet $\left(\mathrm{P}_{2 G S}\right)$ is considered. (b)-(d) Comparison between ML regimes obtained with the FDTW model on the FP laser structure of Fig. 1(d), with the MS-DDE model on the equivalent ring laser structure shown in Fig. 1(c) and with a standard DDE approach on the equivalent ring laser structures shown in Fig. 1(a) [7]-[12] and in Fig. 1(b), respectively. Average output power (b), pulse width (c), and peak power (d) are shown as a function of the current above threshold. Simulations of the structure shown in Fig. 1(a), performed with the standard DDE model, show a continuous wave operating regime over the whole range of investigated currents.

equivalent ring laser structure (Fig. 1c). As expected from the above considerations, the threshold current density is equal in the two structures i.e. the threshold current $I_{t h}$ obtained with the MS-DDE is twice the one obtained from the FDTW model simulations. Furthermore, in absence of gain saturation, the differential efficiency of the light-current characteristic should be exactly the same in the two cases; a slight difference is instead observed due to the different gain saturation induced by the field within the FP cavity and in the corresponding equivalent ring cavity. Despite this slight difference, from the above discussion it clearly appears that the comparison between the FDTW and the MS-DDE modelling results can be established by comparing the dynamic regimes in the two structures at the same value of current above threshold $I-I_{t h}$.

Considering a reversely biased SA section, we then performed simulations of ML using both approaches. A fixed $-7 \mathrm{~V}$ SA voltage is considered. Fig. $4 \mathrm{~b}-\mathrm{d}$ show a comparison between results obtained with the FDTW model when applied to the simulation of the FP laser and the corresponding results obtained with the MS-DDE model on the equivalent ring laser depicted in Fig. 1c. Moreover, in the same figure, simulation results obtained using a standard DDE approach are also shown.

Applying the standard DDE approach [7]-[12] to the simulation of the equivalent ring structure depicted in Fig. 1a, a $\mathrm{CW}$ operating regime has been obtained in the whole range of investigated currents. This can be clearly justified by the fact that the SA section is located just after the reference section where the cumulative non-saturable losses of the whole cavity are assumed to be localized. The optical power crossing the

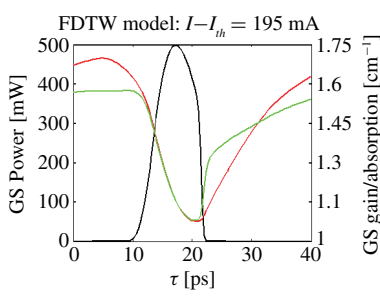

(a)

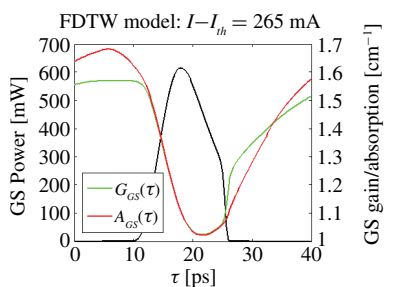

(c)

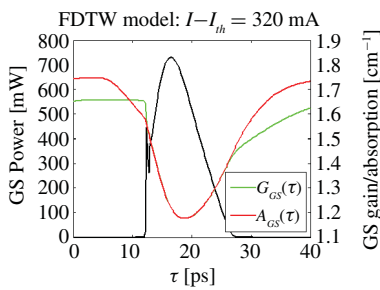

(e)

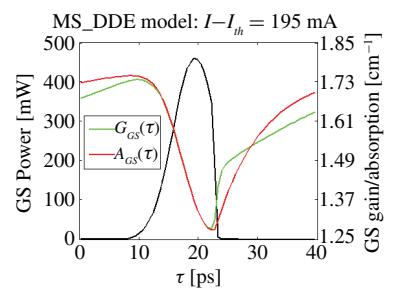

(b)

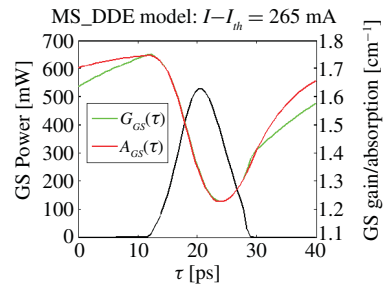

(d)

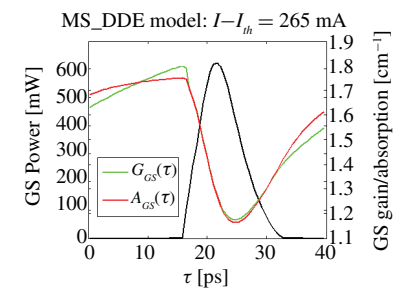

(f)
Fig. 5. Net gain windows leading to the onset of the ML regime in the FP ML laser and in the equivalent ring laser structure of Fig. 1(c) computed using the MS-DDE model (right) and the FDTW model (left) for a 7V SA reverse bias: pulse time trace (blue), $A_{G S}(\tau)$ (red) and $G_{G S}(\tau)$ (green) are shown for currents above threshold of (a), (b) $195 \mathrm{~mA}$, (c), (d) $265 \mathrm{~mA}$, and (e), (f) $320 \mathrm{~mA}$.

SA section is in this case too small to induce a significant absorption bleaching and therefore a stable ML regime cannot be obtained. An alternative choice is to apply the standard DDE approach as shown in Fig. 1b, where the SA section is located before the reference section $z=0$. In this case, a ML regime has been obtained in the whole range of bias currents; however, the average power is significantly smaller and the pulse width is much shorter than those obtained in the FP ML laser, via the FDTW simulations. A significantly improved agreement with the FDTW modelling results can instead be obtained by applying the MS-DDE model to the equivalent ring structure depicted in Fig. 2c. With respect to the previous cases, the difference in the slope of the lightcurrent characteristics obtained with the two methods is now appreciably reduced even if it remains significant, moreover a good agreement in the behaviour of the pulse autocorrelation width versus current is achieved, leading also to an improved quantitative agreement in the values of peak power. From the above considerations, it becomes evident the effectiveness of the proposed MS-DDE model when applied to the simulation of FP ML lasers.

As in the previous section, the net gain windows leading to the ML regimes are investigated. In Fig. 5, we compare the dynamics of the overall saturable and non-saturable cavity losses $A_{G S}(\tau)$, and the overall amplification $\mathrm{G}_{G S}(\tau)$ experienced by the pulse over a round trip in the FP ML laser and in the equivalent unidirectional ring laser of Fig. 2c, computed 


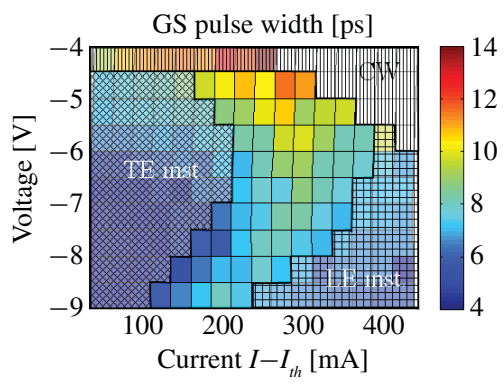

(a)

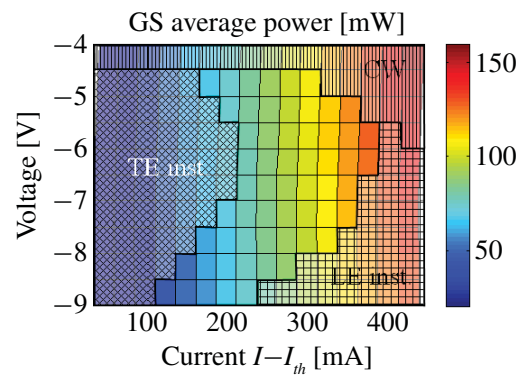

(b)

Fig. 6. Maps of (a) pulse width and (b) average power as a function of the SA reverse voltage and the current above threshold applied to the gain section. Results are obtained with the MS-DDE model from the simulation of the equivalent ring-laser structure shown in Fig. 1(c). Region of continuous wave $(\mathrm{CW})$ operation as well as stability boundaries for the ML regime due to instabilities at the pulse trailing edge (TE inst.) and at the pulse leading edge (LE inst.) are highlighted.

using the FDTW model and MS-DDE model respectively. A general agreement between the net gain windows obtained with the two approaches is preserved: as already shown in Fig. 3, at low currents a net gain window exceeding beyond the pulse trailing edge is obtained; for currents close to threshold, this effect is responsible for instabilities in the ML regime due to noise perturbations. Increasing the current a regime of perfectly stable ML according to New's condition [5], [6], [15] is achieved; finally, at very high gain currents an instability at the pulse leading edge starts to appear. Despite this general agreement, clear differences between the net gain windows obtained with the FDTW model and the MS-DDE model are however present. These discrepancies arise directly from intrinsic differences between the structures simulated with the two approaches. In the FP ML laser simulated with the FDTW model, the cavity losses $A_{G S}(\tau)$ tend to decrease even before the onset of the ML pulse; in the ring laser instead this effect is completely absent. This behaviour is related to the coupling between forward and backward travelling fields in the HR coated SA section: the part of the pulse which has been reflected back by the HR coated facet sees an absorption which has been already partially bleached by the pulse itself when travelling in the SA before being reflected. The coupling between forward and backward travelling fields also partially influences the dynamics of $\mathrm{G}_{G S}(\tau)$.

The study of the net gain windows allows to define stability boundaries for the fundamental ML regime. In Fig. 6, we show maps of pulse width and average power as a function of SA reverse bias and gain current, obtained with the MS-DDE model, where ML stability boundaries are highlighted.

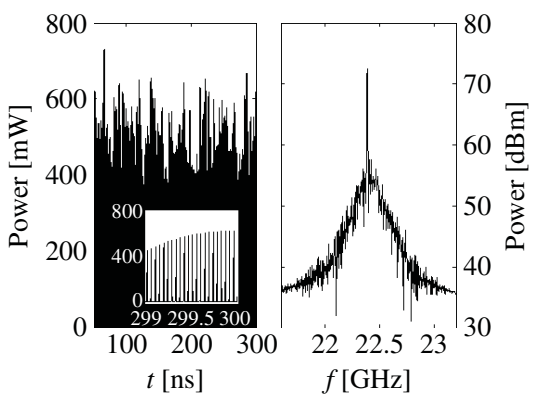

(a)

(b)

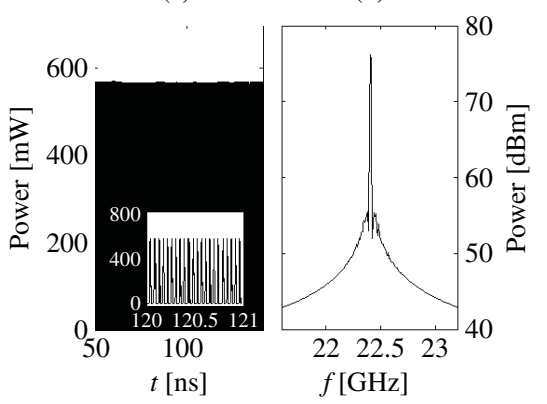

(c)

(d)

Fig. 7. Time traces of the ML pulses and radio frequency spectra computed using the FDTW model, including spontaneous emission noise for a fixed -7 V SA voltage and current (a), (b) $I-I_{t h}=62 \mathrm{~mA}$ and (c), (d) $I-I_{t h}=$ $265 \mathrm{~mA}$.

In the results shown above spontaneous emission noise was not included in the calculation. As shown in [3] however, introducing spontaneous emission (SE) in the calculations, the effectiveness of the noise in deteriorating the ML properties due to either trailing edge or leading edge instabilities can be clearly proved. As an example, in Fig. 7, we show the pulse time traces and the corresponding radio-frequency (RF) spectra, calculated via the FDTW model including SE [3], for a $-7 \mathrm{~V}$ SA voltage and $I-I_{\text {th }}$ equal to $62 \mathrm{~mA}$ and $265 \mathrm{~mA}$, respectively. The large region of net gain extending beyond the pulse trailing edge identified at low currents induces a strong instability in the ML regime that can be clearly seen both in the ML time trace and in the RF spectrum (Fig. 7a-b).

Despite the lack of a precise quantitative agreement, thanks to its very low computational cost, the newly proposed MSDDE model allows to perform extensive quantitative analysis of the ML regimes in FP ML lasers as a function of the bias parameters. The MS-DDE model represents therefore a powerful and computationally efficient approach for the analysis, design and optimization of the performances of FP QD ML lasers. A reduced set of simulations performed using the FDTW model can then be done for validation and refinement of the results obtained with the MS-DDE approach.

\section{CONClusion}

We proposed a new multi-section DDE model for the simulation of passive mode-locking in QD lasers. Its application to the simulation of unidirectional ring lasers has been verified to be in good agreement with a FDTW model. In order to simulate ML in Fabry-Perot lasers using the MSDDE approach, we then identified a ring laser structure whose 
properties are equivalent to those of the FP ML laser under study. Simulating this structure with the MS-DDE approach, a widely improved agreement with the FDTW model results is obtained with respect to standard DDE approaches, proving that the MS-DDE model can be a powerful and flexible method to obtain a reliable description of ML lasers with FP cavities.

The MS-DDE model has been applied here to the study of ML lasers based on semiconductor QDs. This approach however has a general validity and ensures an improved description of FP ML lasers even with quantum-well and bulk active media.

\section{REFERENCES}

[1] E. U. Rafailov, M. A. Cataluna, and W. Sibbett, "Mode-locked quantumdot lasers," Nature Photon., vol. 1, pp. 395-401, Jul. 2007.

[2] M. G. Thompson, A. R. Rae, M. Xia, R. V. Penty, and I. H. White, "InGaAs quantum-dot mode-locked laser diodes," IEEE J. Sel. Topics Quantum Electron., vol. 15, no. 3, pp. 661-672, May-Jun. 2009.

[3] M. Rossetti, P. Bardella, and I. Montrosset, "Time-domain travellingwave model for quantum dot passively mode-locked lasers," IEEE $J$. Quantum Electron., to be published.

[4] A. G. Vladimirov, A. S. Pimenov, and D. Rachinskii, "Numerical study of dynamical regimes in a monolithic passively mode-locked semiconductor laser," IEEE J. Quantum Electron., vol. 45, no. 5, pp. 462-468, May 2009.

[5] G. New, "Pulse evolution in mode-locked quasi-continuous lasers," IEEE J. Quantum Electron., vol. 10, no. 2, pp. 115-124, Feb. 1974.

[6] H. Haus, "Theory of mode locking with a slow saturable absorber," IEEE J. Quantum Electron., vol. 11, no. 9, pp. 736-746, Sep. 1975.

[7] A. G. Vladimirov and D. Turaev, "Model for passive mode locking in semiconductor lasers," Phys. Rev. A, vol. 72, no. 3, pp. 033808-1033808-13, Sep. 2005.

[8] A. G. Vladimirov, D. Turaev, and G. Kozyreff, "Delay differential equations for mode-locked semiconductor lasers," Opt. Lett., vol. 29, no. 11, pp. 1221-1223, Jun. 2004.

[9] E. A. Viktorov, P. Mandel, A. G. Vladimirov, and U. Bandelow, "Model for mode locking in quantum dot lasers," Appl. Phys. Lett., vol. 88, no. 20, pp. 201102-1-201102-3, May 2006.

[10] M. A. Cataluna, E. A. Viktorov, P. Mandel, W. Sibbett, D. A. Livshits, J. Weimert, A. R. Kovsh, and E. U. Rafailov, "Temperature dependence of pulse duration in a mode-locked quantum-dot laser," Appl. Phys. Lett., vol. 90, no. 10, pp. 101102-1-101102-3, Mar. 2007.

[11] E. A. Viktorov, P. Mandel, M. Kuntz, G. Fiol, D. Bimberg, A. G Vladimirov, and M. Wolfrum, "Stability of the mode-locked regime in quantum dot laser," Appl. Phys. Lett., vol. 91, no. 23, pp. 231116-1231116-3, Dec. 2007.
[12] A. G. Vladimirov, U. Bandelow, G. Fiol, D. Arsenijević, M. Kleinert, D. Bimberg, A. Pimenov, and D. Rachinskii, "Dynamical regimes in a monolithic passively mode-locked quantum dot laser," J. Opt. Soc. Am. $B$, vol. 27, no. 10, pp. 2102-2109, 2010.

[13] U. Bandelow, M. Radziunas, A. G. Vladimirov, B. Hüttl, and R. Kaiser, "40 GHz mode-locked semiconductor lasers: Theory, simulations and experiment," Opt. Quantum Electron., vol. 38, nos. 4-6, pp. 495-512, 2006.

[14] M. Gioannini and I. Montrosset, "Numerical analysis of the frequency chirp in quantum-dot semiconductor lasers," IEEE J. Quantum Electron., vol. 43, no. 10, pp. 941-949, Oct. 2007.

[15] R. G. M. P. Koumans and R. van Roijen, "Theory for passive modelocking in semiconductor laser structures including the effects of selfphase modulation, dispersion, and pulse collisions," IEEE J. Quantum Electron., vol. 32, no. 3, pp. 478-492, Mar. 1996.

Mattia Rossetti received the Laurea degree in physical engineering in 2007 from the Politecnico di Torino, Turin, Italy, where he is currently working toward the Ph.D. degree in electronic and communication engineering.

$\mathrm{He}$ is currently with the Dipartimento di Elettronica, Politecnico di Torino. His current research interests include the simulation of quantum-dot-based optoelectronic devices.

Paolo Bardella received the Laurea degree in electronic engineering and the Ph.D. degree in electronic and communication engineering from the Politecnico di Torino, Turin, Italy, in 2001 and 2006, respectively.

He is currently a Post-Doctoral Fellow with the Dipartimento di Elettronica, Politecnico di Torino, where he works on the simulation of multisection semiconductor lasers in mode-locking and self-pulsating regime. His current research interests include modeling and design of multisection quantum-dot semiconductor lasers and super-luminescent diodes.

Ivo Montrosset (M'92) was born in Aosta, Italy, in 1946. He received the Laurea degree in electronic engineering from the Politecnico di Torino, Turin, Italy, in 1971.

He is currently with the Dipartimento di Elettronica, Politecnico di Torino. From 1972 to 1986, he was with the Politecnico di Torino. In 1986, he was appointed Full Professor at the Università di Genova, Genova, Italy. Since 1990, he has been Full Professor of optoelectronics at Politecnico di Torino. His main research interests include guided-wave optics, solid-state and semiconductor lasers, and other related topics.

Prof. Montrosset is a member of the IEEE Photonics Society. 